Association for Information Systems AIS Electronic Library (AISeL)

Wirtschaftsinformatik Proceedings 1999

Wirtschaftsinformatik

February 1999

\title{
Dynamische Marktmodelle im elektronischen Wertpapierhandel
}

Miroslav Budimir

UniversitätGießen, miroslav.budimir@wirtschaft.uni-giessen.de

Peter Gomber

UniversitätGießen, peter.gomber@wirtschaft.uni-giessen.de

Follow this and additional works at: http://aisel.aisnet.org/wi1999

\section{Recommended Citation}

Budimir, Miroslav and Gomber, Peter, "Dynamische Marktmodelle im elektronischen Wertpapierhandel" (1999). Wirtschaftsinformatik Proceedings 1999. 15.

http://aisel.aisnet.org/wi1999/15

This material is brought to you by the Wirtschaftsinformatik at AIS Electronic Library (AISeL). It has been accepted for inclusion in Wirtschaftsinformatik Proceedings 1999 by an authorized administrator of AIS Electronic Library (AISeL). For more information, please contact elibrary@aisnet.org. 


\section{Dynamische Marktmodelle im elektronischen Wertpapierhandel}

Miroslav Budimir

Universität Gießen (miroslav.budimir@ wirtschaft.uni-giessen.de)

Peter Gomber

Universität Gießen (peter.gomber@wirtschaft.uni-giessen.de)

\section{Inhalt}

1 Einleitung

2 Marktmodelle der Gegenwart

3 Anforderungen an Marktmodelle

3.1 Handel in nicht standardisierten Produkten

3.2 Illiquide Wertpapiere

3.3 Blockhandel

4 Dynamische Marktmodelle als Lösungsansatz

4.1 Dynamische Marktmodelle

4.2 Softwareagenten

5 Umsetzung eines dynamischen Marktmodells - das System AMTRAS

6 Zusammenfassung und Ausblick 


\begin{abstract}
Die Prozesse und Regeln, die Angebot und Nachfrage auf Kapitalmärkten zusammenführen, stellen einen kritischen Erfolgsfaktor für Wertpapierbörsen dar. Die Heterogenität der Marktteilnehmer spiegelt sich in heterogenen Anforderungen an diese Regeln, die als Marktmodell bezeichnet werden, wider. Gegenwärtig existiert eine Vielzahl von Börsen mit unterschiedlichen Marktmodellen, so daß die Marktteilnehmer durch die Wahl des Börsenplatzes bzw. -systems auch das für ihre Transaktionsinteressen vorteilhafte Regelwerk auswählen können.
\end{abstract}

Die Tendenz zu Kooperationen zwischen Börsen sowie der Wegfall traditioneller Handelsformen auf dem Parkett führt zur Abnahme der Anzahl an Handelsplattformen und damit der Anzahl alternativer Marktmodelle. Dies reduziert die Auswahlmöglichkeiten und bedeutet damit einen Qualitätsverlust für die Marktteilnehmer. Aus diesem Grund wird in der Arbeit ein neues Konzept zum Design von Marktmodellen entwickelt. Damit sollen die verschiedenartigen Anforderungen der Handelsteilnehmer mittels einer elektronischen Handelsplattform erfüllt werden, indem die Marktteilnehmer - je nach Transaktionswunsch - selbst das jeweilige Marktmodell bestimmen können.

Dazu wird in diesem Beitrag das Konzept dynamischer Marktmodelle entwickelt und den Anforderungen der Marktteilnehmer gegenübergestellt. Zur softwaretechnischen Umsetzung dieses Konzeptes werden die Charakterisierungsmerkmale des Paradigmas Intelligenter Agenten herausgearbeitet und gezeigt, daß mit Hilfe dieser Technologie ein dynamisches Marktmodell realisiert werden kann.

Schließlich wird das agentenbasierte elektronische Wertpapierhandelssystem AMTRAS vorgestellt. Dabei handelt es sich um die konkrete Umsetzung eines dynamischen Marktmodells für den Rentenmarkt.

\title{
1 Einleitung
}

Neue Medien und zukunftsweisende Kommunikationsformen haben in den letzten Jahren wesentlich zur Entstehung einer Vielzahl Elektronischer Märkte in verschiedenen Produkt- und Dienstleistungsbereichen beigetragen. Darüber hinaus haben die neuen Möglichkeiten der Informationstechnologie und der damit verbundene Innovationsschub auch zu fundamentalen Änderungen bereits etablierter Marktstrukturen geführt. Hier sind insbesondere die Wertpapiermärkte zu nennen, deren - zum Teil über viele Jahrzehnte gewachsene - Strukturen und Organisationsformen sich in den letzten Jahren fundamental gewandelt haben. 
Als Gründe hierfür sind neben dem Einfluß der Informationstechnologie auch die wachsende Bedeutung institutioneller Investoren sowie eine nachhaltige Deregulierung der Märkte zu nennen, die einen stärkeren Wettbewerb der Börsen sowie die Einführung von Finanzinnovationen ermöglichte (Modigliani und Fabozzi 1992, S. 225).

Zeitgemäße Börsen sind ohne elektronische Unterstützung in den Transaktionsphasen Ordereingabe, Orderzusammenführung, Clearing und Information nicht mehr denkbar (Gerke 1993). Auch eine vollständige Substitution der Parkettbörsen durch elektronische Handelssysteme (EHS) ist in naher Zukunft nicht auszuschließen. Das beste Beispiel hierfür stellt die vollelektronische Terminbörse Eurex ${ }^{1}$ dar, die im wichtigen Marktsegment der BUND-Futures nunmehr nach langer Dominanz der Londoner Parkettbörse LIFFE die Marktführerschaft erlangt hat. Elektronische Handelsplattformen mit dezentralem Marktzugang ohne Erfordernis einer physischen Präsenz am Handelsplatz ermöglichen den Aufbau internationaler Börsennetze, wie etwa die angekündigte Kooperation zwischen der Deutschen Börse AG und der London Stock Exchange (Deutsche Börse 1998a).

Unabhängig von der Organisation als Parkett- oder Computerbörse ist die Art des Marktmodells, d. h. das Regelwerk der Marktorganisation nach dem Angebot und Nachfrage zusammengeführt werden, ein zentraler Erfolgsfaktor jeder Börse. Die Kooperation von Börsen und im Extremfall deren Verschmelzung führen jedoch zu einer Verringerung der Anzahl alternativer Marktmodelle. Die unterschiedlichen Anlegergruppen stellen unterschiedliche Anforderungen an Marktmodelle und profitieren von der Vielzahl der gegenwärtig angebotenen Marktmodelle, indem sie sich - abhängig von der Art des aktuellen Transaktionswunsches - für einen bestimmten Börsenplatz oder sogar für außerbörsliche Handelsformen entscheiden. Beispielsweise bevorzugen große Marktteilnehmer den Handel ohne die Einschaltung von Intermediären auf einer kostengünstigen und transparenten elektronischen Plattform. Ein Marktmodell mit Kursmaklern hingegen kann die Übervorteilung von Privatanlegern durch Berufshändler - welche die Orderlage kennen und flexibel reagieren können ausschließen, da der Kursmakler verpflichtet ist, Kurse nach der ,wirklichen Geschäftslage“ zu stellen. Eine Reduktion von Marktmodellen bedeutet eine Verringerung der Wahlmöglichkeiten der Anleger und ist somit einem Qualitätsverlust gleichzusetzen.

Die Marktteilnehmer stellen hohe Ansprüche an die zugrundeliegenden Marktmodelle der Börsen. Die Börsen hingegen versuchen aus Kosten- und Effizienzgründen, den Marktteilnehmern einfache und weitgehend standardisierte Marktmodelle zur Verfügung zu stellen, um somit Economies of scale nutzen zu können. Es stellt somit eine Herausforderung dar, dieses Spannungsfeld aufzu-

1 Bis zum 19. Juni 1998 firmierte die heutige Eurex unter dem Namen DTB - Deutsche Terminbörse (o. V. 1998). 
lösen und Investoren die Vorteile einer Vielzahl zur Verfügung stehender Marktmodelle auch dann zu bieten, wenn die Anzahl der Handelsalternativen durch Kooperationen und Mergers oder durch die Aufgabe des dualen Handels d. h. der gleichzeitigen Existenz von Parkett- und Computerhandel in einer Börseninstitution - abnimmt.

Vor diesem Hintergrund untersucht die Arbeit einen Lösungsansatz zur Auflösung dieses Spannungsfeldes. Dabei sollen innovative Marktmodelle gefunden werden, die einerseits den Marktteilnehmern die Möglichkeit bieten, für jede Transaktion aus mehreren Alternativen ein gewünschtes Marktmodell auszuwählen, andererseits jedoch den Börsen ermöglichen, auf einer einzigen elektronischen Plattform mehrere Marktmodelle alternativ bereitzustellen. Dazu werden auf der Basis einer Analyse bestehender Marktmodelle die Anforderungen der verschiedenen Investorengruppen herausgearbeitet und das Konzept der dynamischen Marktmodelle eingeführt.

Aus technischer Sicht erfordert die Umsetzung dynamischer Marktmodelle eine hohe Flexibilität des zugrundeliegenden Softwareparadigmas. In diesem Zusammenhang wird gezeigt, welches Potential die Technologie der Softwareagenten zur Erfüllung dieser Anforderungen bietet. Zur prototypischen Umsetzung des Konzeptes dynamischer Marktmodelle wurde das System AMTRAS (Agent Mediated Trading System) entwickelt. AMTRAS stellt ein dynamisches Marktmodell auf der Basis agentenbasierter Technologie zur Verfügung.

Im nächsten Abschnitt werden der Begriff des Marktmodells und verschiedene existierende Ausprägungen näher erläutert. Die Analyse der Anforderungen verschiedener Investorengruppen im dritten Abschnitt führt zur Spezifikation des Konzeptes des dynamischen Marktmodells. Abschnitt 4 arbeitet die besonderen Vorteile des Paradigmas der Softwareagenten für die Umsetzung dieses Konzeptes heraus. Im fünften Abschnitt wird das System AMTRAS beschrieben. Eine Zusammenfassung und ein Ausblick auf die weitere Forschungsarbeit schließen die Arbeit ab.

\section{Marktmodelle der Gegenwart}

Der Begriff des Marktmodells wird in der Literatur nicht einheitlich definiert. Die Deutsche Börse (Deutsche Börse 1998b, S. 7) beispielsweise versteht den Begriff Marktmodell als "den Mechanismus, auf welche Weise Orders zu Geschäftsabschlüssen im börslichen Handelssystem zusammengeführt werden." Gerke und Rapp (1994) hingegen bedienen sich einer allgemeineren Definition: ein Marktmodell wird als "das aus technischen, organisatorischen, rechtlichen und gewohnheitsrechtlichen Handelsregeln bestehende Strukturgerüst eines institutionalisierten Börsenhandels" angesehen. 
Weltweit existiert eine Vielzahl unterschiedlicher Wertpapierbörsen und somit auch eine Vielzahl von Marktmodellen. Diese unterscheiden sich in ihren Strukturmerkmalen (Lüdecke 1996), beispielsweise dem Verfahren der Preisermittlung, der Rolle der Intermediäre beim Wertpapierhandel, der Handelsfrequenz sowie in der Art und dem Umfang der verfügbaren Informationen. Jedes dieser Strukturmerkmale kann mehrere Ausprägungen annehmen. So kann z.B. das Merkmal "Verfahren der Preisermittlung" durch die Ausprägungen "Preisermittlung nach dem Auktionsprinzip" oder "Preisermittlung nach dem MarketMaker-Prinzip" gekennzeichnet sein. Abbildung 1 führt wesentliche Strukturmerkmale und mögliche Ausprägungen beispielhaft auf. Legt man konkrete Ausprägungen für jedes Strukturmerkmal fest, gelangt man zu einem konkreten Marktmodell.

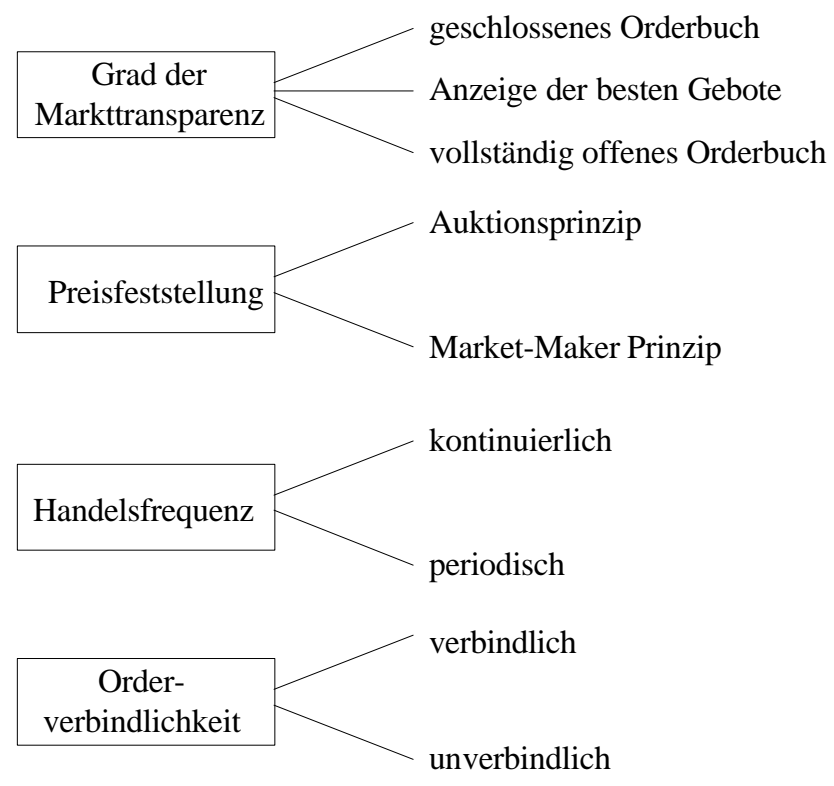

Abbildung 1: Strukturmerkmale und mögliche Ausprägungen

Die oben aufgeführten Definitionen sind für die Zwecke der vorliegenden Arbeit etwas zu weit gefaßt. Daher ist es notwendig, eine engere Definition vorzuschlagen und sich dabei auf Strukturmerkmale zu konzentrieren. Demzufolge ist ein Marktmodell als eine Kombination aller für den Börsenhandel relevanten Ausprägungen börslicher Strukturmerkmale charakterisiert. In Abhängigkeit von den einzelnen Kombinationsmöglichkeiten der Strukturmerkmale werden hier statische und flexible Marktmodelle unterschieden.

Statische Marktmodelle liegen vor, wenn für jedes Strukturmerkmal nur eine Ausprägung zugelassen wird. Beispielsweise liegt bei vielen weniger liquiden 
Wertpapieren, für die lediglich eine Einheitskursfeststellung am Parkett erfolgt, eine statische Marktstruktur vor, da als Preisermittlungsverfahren nur die Methode der - periodisch stattfindenden - Auktion in Frage kommt. Statische Marktmodelle sind die einfachste Form eines organisierten Börsenhandels: Bei dieser Art von Marktmodellen erfolgt die Orderzusammenführung nur nach einer im voraus festgelegten Regel. Der Handel an solchen Märkten ist inflexibel und nicht an heterogene Anforderungen vieler Handelsteilnehmer angepaßt. Deshalb existieren heute flexible Marktmodelle.

Ein flexibles Marktmodell liegt vor, wenn für mindestens ein Strukturmerkmal mehrere Ausprägungen möglich sind. Diese Ausprägungen müssen allen Marktteilnehmern ex ante bekannt sein. Außerdem sind genaue Regeln festzulegen, unter welchen Bedingungen welche Ausprägungen in Kraft treten. Als Beispiel kann das Marktmodell von Xetra herangezogen werden. Das Strukturmerkmal "Handelsfrequenz" kann bei Xetra zwei Ausprägungen annehmen: in der Phase des fortlaufenden Handels wird kontinuierlich gehandelt, d. h. der Handel findet durch eine Folge von bilateralen Transaktionen zu unterschiedlichen Preisen (Einzelpreisen) statt. Zu definierten Zeitpunkten oder beim Auftreten von bestimmten Bedingungen - wie etwa der Eröffnung und dem Schluß des Handels sowie bei Volatilitätsunterbrechungen - sind Auktionsphasen (Eröffnungs-, Schlußauktion und Auktion während des fortlaufenden Handels) vorgesehen, in denen die im Orderbuch vorhandenen Orders nach dem Meistausführungsprinzip zusammengeführt und zum Einheitspreis ausgeführt werden.

Obwohl flexible Marktmodelle in größerem Maße an die Bedürfnisse der Handelsteilnehmer angepaßt sind als statische, stellen flexible Marktmodelle immer noch einen Kompromiß zwischen den unterschiedlichen Interessen der Marktteilnehmer dar (Braue und Hille 1997). Bisher war es nicht möglich, ein Marktmodell zu entwickeln, welches den Anforderungen einer Vielzahl von Marktteilnehmern gleichzeitig gerecht werden konnte. Zu diesem Zweck wird ein Modell benötigt, welches die einzelnen Handelsteilnehmer individuell an ihre aktuellen Bedürfnisse anpassen können. Im folgenden werden die Anforderungen der Handelsteilnehmer an solche Marktmodelle herausgearbeitet, um darauf aufbauend zu untersuchen, inwiefern die gegenwärtigen Marktmodelle zur Erfüllung dieser Anforderungen geeignet sind.

\section{Anforderungen an Marktmodelle}

Schwächen der gegenwärtigen Marktmodelle der Börsen werden vor allem im Handel mit solchen Wertpapieren offenbar, die hauptsächlich außerbörslich - am Over the counter-Markt (OTC) - gehandelt werden. Dabei handelt es sich im wesentlichen um (Weinhardt und Gomber 1998):

- Wertpapiere, die aufgrund fehlender Standardisierung nicht an Börsen gehandelt werden, wie etwa derivative Finanzinstrumente, 
- illiquide Wertpapiere, d. h. Papiere, die nur mit einem großen Preisab- bzw. aufschlag gehandelt werden können, wie z.B. Pfandbriefe oder Aktien kleiner Unternehmen und

- Handel in großen Volumina (Blockhandel), der einen - meist temporären adversen Preiseffekt hervorrufen kann.

Solche Transaktionen werden von Händlern hauptsächlich am OTC-Markt und nicht an der Börse gehandelt, da die zugrundeliegenden Marktmodelle der Börsen die Anforderungen der Händler nicht erfüllen. Im folgenden wird einzeln auf die drei genannten Problembereiche eingegangen.

\subsection{Handel in nicht standardisierten Produkten}

An Börsen werden standardisierte Verträge nach genau festgelegten Usancen und von eigens hierzu befähigten Marktteilnehmern geschlossen (Büschgen 1993, S. 112). Wertpapiere ohne hohen Standardisierungsgrad können an Börsen nicht gehandelt werden, da die Ausgestaltungsmerkmale dieser Kontrakte individuell zwischen den Verhandlungspartnern vereinbart werden und bei jedem Abschluß unterschiedlich ausgestaltet sein können. Hier seien beispielsweise Zins-Derivate wie Forwards, Swaps, Caps, Floors, Swaptions usw. genannt.

Die Marktmodelle existierender Börsen ermöglichen keinen Handel in nicht standardisierten Produkten. Vor allem ein elektronischer Handel solcher Kontrakte ist bislang nicht realisiert, da die gegenwärtigen EHS nur für den Handel solcher Wertpapiere konzipiert sind, die durch eindeutige Merkmale gekennzeichnet und über eine eindeutige Wertpapierkennummer (WKN) identifiziert werden. Daher ist ein Marktmodell $\mathrm{zu}$ konzipieren, welches die Verhaltensweisen der Händler am OTC-Markt bei der Verhandlung einzelner Vertragskomponenten hinreichend genau abbildet. Dabei spielt nicht nur der Prozeß der Preisfindung eine Rolle, sondern auch der Prozeß der Suche nach einem geeigneten Produkt.

Diese Produktsuche kann dadurch abgebildet werden, daß Händler bei der Angabe ihres Transaktionswunsches nicht ein konkretes Wertpapier spezifizieren müssen, sondern auch unscharf die von ihnen gewünschten Ausprägungen der Produktparameter angeben können. Die Händler sind in der Lage, bei einem solchen Marktmodell eine beliebige Anzahl von Parametern zu spezifizieren, wobei die Wahrscheinlichkeit des Matchings - also des Auffindens eines entsprechenden Wertpapiers auf der gegenüberliegenden Marktseite - mit der Anzahl der gewünschten Parameter abnimmt. Es können beispielsweise folgende Parameter eingegeben werden:

- Coupon (bei festverzinslichen Wertpapieren). Hier ist eine exakte Angabe (z.B. genau 5\%) oder das Aufführen einer gewünschten Bandbreite (z.B. 5\% bis $5,75 \%$ ) möglich.

- Restlaufzeit (bei festverzinslichen Wertpapieren). Auch hier ist es möglich, eine genaue Restlaufzeit anzugeben (z.B. 10 Jahre) oder ein Restlaufzeitintervall (z.B. 5 bis 7 Jahre) zu spezifizieren. 
- Referenzzinssatz (bei Caps). Händler können beliebige Referenzzinsätze spezifizieren, z.B. 3-Monats-LIBOR (London Interbank Offered Rate), 6Monats-LIBOR oder EURIBOR (European Interbank Offered Rate).

- Kontraktbeginn (bei Forwards). Ähnlich wie bei der Restlaufzeit kann hier ein exakter Wert oder eine Bandbreite angegeben werden.

- Basis (bei OTC-Optionen). Beim Handel von Optionen müssen die Händler eine konkrete Basis sowie den Basispreis vereinbaren. Nach Festlegung der Basis kann der gewünschte Basispreis - entsprechend der obengenannten Vorgehensweise - entweder genau spezifiziert oder in einer Bandbreite festlegt werden.

Ein innovatives Marktmodell sollte also den Marktteilnehmern auch die Möglichkeit bieten, unscharfe Produktparameter spezifizieren zu können, um auf dieser Basis individuelle Kontrakte auszuhandeln.

\subsection{Illiquide Wertpapiere}

Es existiert eine große Anzahl von Wertpapieren, die ohne größere Preisauf- bzw. abschläge nicht sofort ge- bzw. verkauft werden können. Dabei handelt es sich um illiquide Wertpapiere (Brunner 1996, S. 4-6). Als Beispiele können hier Aktien kleinerer Unternehmen sowie Pfandbriefe mit geringen Emissionsvolumina genannt werden. Als Möglichkeit zur Erhöhung der Matchingwahrscheinlichkeit bietet sich bei der Produktsuche die oben beschriebene, unscharfe Suche nach Parametern an.

Beim gegenwärtigen außerbörslichen Telefonhandel ist für einen Händler der Kreis seiner potentiellen Abschlußpartner auf die Anzahl seiner Telefonpartner beschränkt. Er kann bei seiner Partnersuche somit nur einen kleinen Ausschnitt des aktuellen Marktes erreichen. Der Händler kann seinen Suchraum auf zwei Arten vergrößern: Entweder durch die Beauftragung eines Brokers oder durch selbständige Erhöhung der Anzahl seiner telefonischen Ansprechpartner. Beide Möglichkeiten beinhalten jedoch Nachteile: Die Einschaltung von Brokern verursacht hohe Transaktionskosten. Eine höhere Anzahl von Kontraktpartnern stößt auf technische Schwierigkeiten, da der Händler nicht in der Lage ist, mit einer beliebig großen Anzahl von Partnern telefonisch simultan oder sukzessive $\mathrm{zu}$ verhandeln. Insbesondere bei illiquiden Papieren sollte das Marktmodell ermöglichen, den Suchraum individuell und flexibel zu vergrößern und potentielle Partner parallel $\mathrm{zu}$ adressieren. Dies kann die Liquidität positiv beeinflußen.

Ein weiteres Argument für ein Marktmodell, welches eine individuelle Einstellung des Partnersuchraumes zuläßt, kann mit dem Ausfall- und Wiederbeschaffungsrisiko erklärt werden (Weinhardt und Gomber 1998). Ein Händler ist daran interessiert, mit solchen Partnern Geschäfte zu tätigen, bei denen er bezüglich der Erfüllung sicher sein kann. Aus diesem Grund bietet sich - ähnlich einer Abstufung der Händler in Bonitätsklassen - eine mehrstufige Kontrahentenauswahl an, wie Abbildung 2 darstellt. 


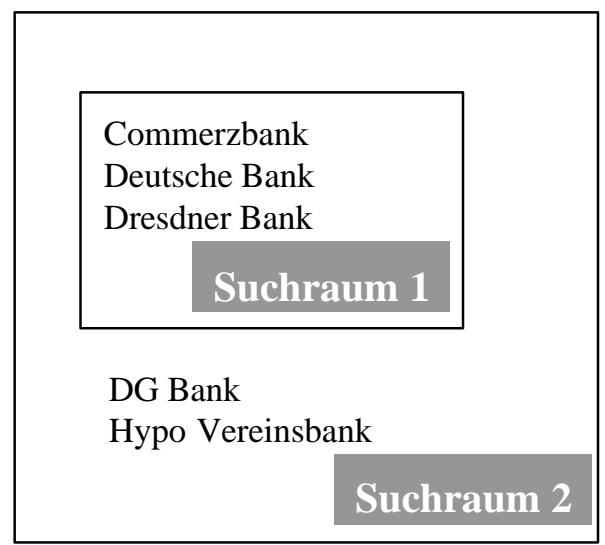

\author{
BHF-Bank \\ Citibank \\ Dresdner Bank

\section{Suchraum 3}

Abbildung 2: Ein Beispiel für Partnersuchräume

Der Unterschied dieser Abstufung zu einem herkömmlichen Rating liegt darin, daß jeder Händler seinen Suchraum individuell auf der Basis seiner Bonitätseinschätzungen konfigurieren kann. Dabei kann er sich an verschiedenen Kriterien orientieren. So können etwa die Qualität der vergangenen Transaktionen mit einem entsprechenden Händler, die von Rating-Agenturen eingeschätzte Bonität des jeweiligen Instituts oder auch persönliche Beziehungen eine Rolle spielen. Ein Kontraktpartner kann dabei in mehreren Suchräumen enthalten sein: In Abbildung 2 befindet sich die Dresdner Bank in allen drei dargestellten Suchräumen. Die Suchräume sollten vom Händler vollkommen frei konfigurierbar sein. Ein Matching findet nur dann statt, wenn beide Händler in ihren Suchräumen den jeweils anderen Partner angegeben haben.

\title{
3.3 Blockhandel
}

Als Blockhandel bezeichnet man Orders größerer Volumina, die ungewünschte Kursveränderungen verursachen können (Gerke und Rasch 1992). Eine mögliche Ursache des adversen Preiseffektes liegt in der Informationsasymmetrie zwischen den Marktteilnehmern. Beim Vorliegen einer Blockorder vermuten die restlichen Händler einen Informationsvorsprung des Blockhändlers: Es wird angenommen, daß bei einer Kauforder (Verkauforder) dem Blockhändler positive (negative) private Informationen über die zukünftige Kursentwicklung des Wertpapiers vorliegen. Deshalb wird der Markt den Block nur dann absorbieren, wenn eine Kompensation für das übernommene Preisänderungsrisiko - in Form von günstigeren Kursen für die andere Marktseite - erfolgt (Easley und O'Hara 1992).

Um solche adverse Preiseffekte zu umgehen, versucht ein Blockhändler, die Transaktionsabsicht vor den restlichen Marktteilnehmern zu verbergen. Er wird vermeiden, eine Order ins offene Buch der Börse einzugeben, da die restlichen 
Marktteilnehmer davon sofort Kenntnis nehmen werden. Auch das Einstellen in ein geschlossenes Orderbuch kann aufgrund fehlender Marktbreite negative Preiseffekte nach sich ziehen (Hasbrouck und Schwartz 1988). Daher bevorzugen Händler bei Blockorders einen gewissen Grad an Intransparenz, um adversen Preiseffekten zu entgehen. Sie ziehen sich oftmals auf gesonderte Marktsegmente zurück (etwa den sog. Upstairs market (Luskin 1988) der New York Stock Exchange oder spezielle Handelssysteme wie z.B. OptiMark (Gomber 1998)) oder betreiben außerbörslichen Handel über die Einschaltung von Brokern. Der heutige Renten- und Devisenhandel wird fast ausschließlich außerbörslich getätigt. Dies liegt daran, daß der Großteil des Volumens von institutionellen Marktteilnehmern umgesetzt wird. Diese handeln meist große Volumina und wickeln ihre Geschäfte häufig telefonisch ab. Nur noch kleinere Kundenorders werden an der Börse ausgeführt (Thießen 1995).

Spezielle Anforderungen an die Markttransparenz haben auch solche Marktteilnehmer, die bewußt in den Marktprozeß eingreifen, um auf diese Weise Kurse verschiedener Aktiva zu beeinflussen. So betreibt die Bundesbank Kurspflege für Bundeswertpapiere und eine gezielte Interventionspolitik am Devisenmarkt (Deutsche Bundesbank 1995, S. 59-62). Folglich sind die Händler der Bundesbank daran interessiert, ihre Präsenz am Markt offenzulegen, um Signalwirkungen zu erzielen.

Hieraus ergibt sich eine weitere Anforderung an das Marktmodell: Handelsteilnehmer sollen selbständig über den Grad der gewünschten Markttransparenz entscheiden können. Dies sollte für jede einzelne Order möglich sein.

\section{Dynamische Marktmodelle als Lösungsansatz}

\subsection{Dynamische Marktmodelle}

Ein dynamisches Marktmodell liegt vor, wenn die Marktteilnehmer die Ausprägungen der Strukturmerkmale aus einer vorgegebenen Menge für einzelne Transaktionen auswählen können.

Analog einem flexiblen Marktmodell kann bei einem dynamischen Marktmodell jedes börsliche Strukturmerkmal mehrere Ausprägungen aufweisen. Der Unterschied eines dynamischen Marktmodells zum flexiblen besteht darin, daß die Handelsteilnehmer selbst die - für die jeweilige Transaktion geltenden konkreten Ausprägungen der Parameter festlegen können. Die Regeln für das Eintreten bestimmter Ausprägungen sind somit nicht fest vorgegeben. Im Extremfall kann jeder Handelsteilnehmer für sich entscheiden, wann er welches Marktmodell beanspruchen will. Beispielsweise kann ein Händler selbständig entscheiden, ob er an einer Auktion oder an einer bilateralen Verhandlung mit 
weiteren Wertpapierhändlern teilnehmen will bzw. ob er am Marktgeschehen anonym oder unter Preisgabe seiner Identität agieren möchte.

Das Matching setzt das gegenseitige Einverständnis der beteiligten Handelspartner bezüglich der relevanten Wertpapierparameter voraus. Das bedeutet, daß die angebotenen bzw. gesuchten Wertpapiere bezüglich aller Parameter - wie z.B. der Wertpapierart, des Emittenten, des Coupons oder der Laufzeit usw. übereinstimmen müssen. Im Konzept des dynamischen Marktmodells müssen auch die ausgewählten Ausprägungen der Strukturmerkmale zueinander kompatibel sein. Beispielsweise kann eine Order nicht ausgeführt werden, wenn einerseits zwar alle Wertpapierparameter einschließlich der zu handelnden Mengen übereinstimmen, andererseits die Händler jedoch unterschiedliche Präferenzen bezüglich der Preisbildung haben. Präferiert ein Händler die Auktion als Preisfindungsform, der andere eine Verhandlung, kommt kein Matching zustande. Ein Matching kann jedoch erfolgen, wenn einer der beiden Händler vollständige Anonymität beim Verhandlungsprozeß wünscht und der andere gewillt ist, diese Anonymität der Gegenpartei in Kauf zu nehmen und seine Identität zu offenbaren.

Die Flexibilität auf der logischen Ebene erfordert für einen elektronischen Handel auch die entsprechende Umsetzbarkeit auf der technischen Ebene. Das Softwareparadigma Intelligenter Agenten bietet hier einen Lösungsansatz. Dieses wird im folgenden kurz vorgestellt sowie bezüglich der spezifizierten Anforderungen an ein dynamisches Marktmodell analysiert.

\subsection{Softwareagenten}

In der Literatur gibt es keine einheitliche Abgrenzung des Begriffes Softwareagent (kürzer: Agent). Allen Definitionen ist jedoch gemein, daß Agenten in speziellen Domänen Aufgaben für ihren Benutzer durchführen und dabei bestimmte Ziele verfolgen (siehe z.B. Maes 1995, S. 108). Ein Agent hat eine eigenständige Wissensbasis (Wooldridge und Jennings 1995) und soll in der Lage sein, Veränderungen seiner Umgebung wahrzunehmen und sein Verhalten dementsprechend anzupassen. Dies erfordert ein hohes Maß an Autonomie und Flexibilität. Des weiteren muß ein Softwareagent mit dem Benutzer oder mit anderen Agenten kommunizieren können, um die ihm übertragenen Aufgaben erfolgreich zu erfüllen.

Abbildung 3 führt die Charakterisierungsmerkmale von Softwareagenten auf und stellt sie den oben herausgearbeiteten Anforderungen an ein modernes, dynamisches Marktmodell gegenüber. Auf dieser Basis wird geprüft, inwiefern die Technologie der Softwareagenten geeignet ist, ein elektronisches Handelssystem softwaretechnisch abzubilden, welches diese Anforderungen erfüllen kann. 


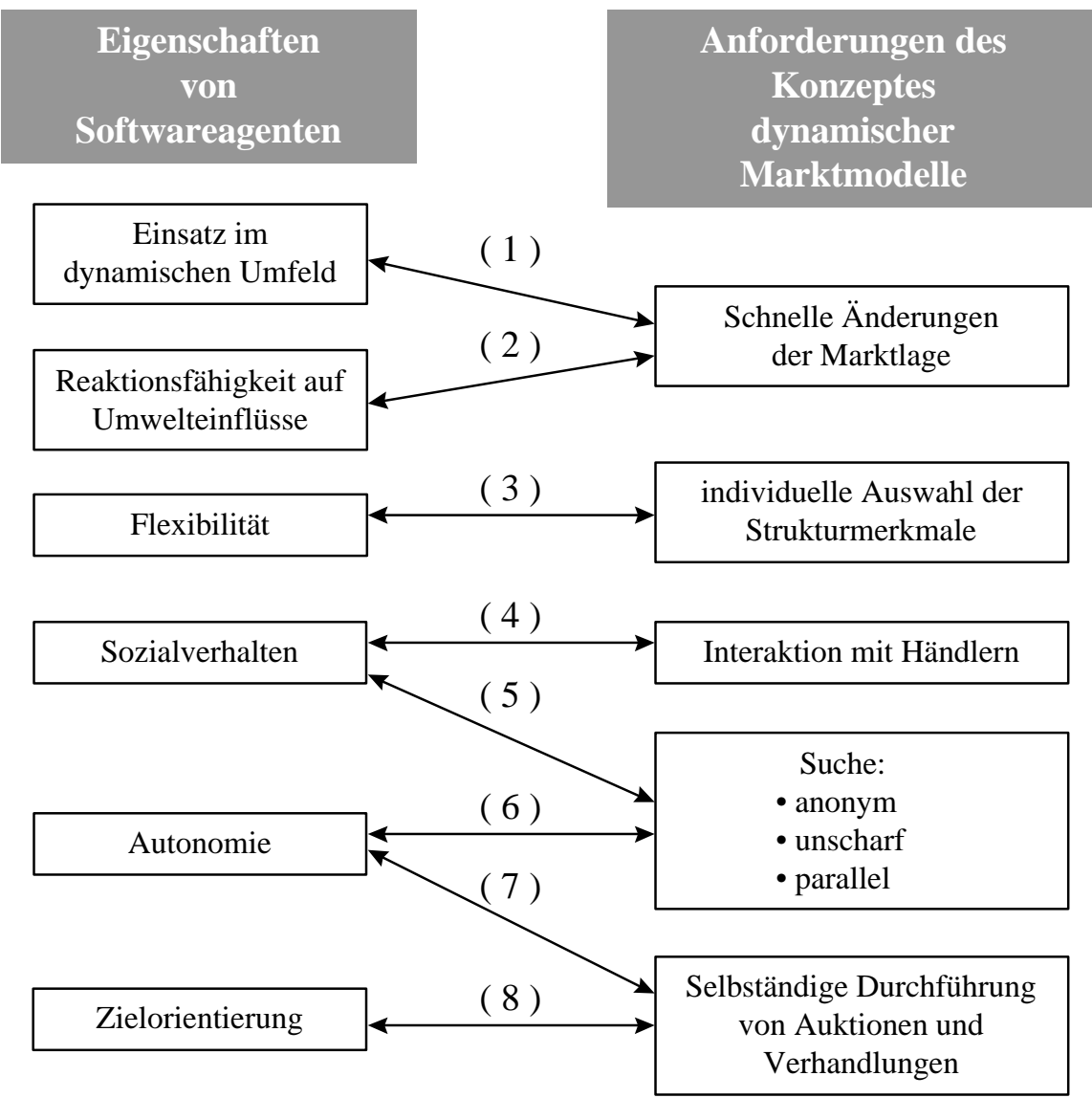

\section{Abbildung 3: Eignung der Technologie der Softwareagenten für dynamische Marktmodelle}

Agenten "leben" in einem komplexen, dynamischen Umfeld (Maes 1995). Die Situation auf Wertpapiermärkten repräsentiert solch ein Umfeld (1). Die Marktteilnehmer erhalten über Nachrichtendienste wie Reuters oder Bloomberg unentwegt neue Informationen, die den Kurs des betrachteten Wertpapiers beeinflussen können. Händler müssen das Marktgeschehen stets beobachten und die von ihnen gehaltenen Positionen laufend der aktuellen Marktsituation anpassen. Agenten können selbständig Änderungen ihrer Umwelt wahrnehmen und darauf entsprechend reagieren (2). Diese Eigenschaft kann ein Händler beispielsweise dazu verwenden, den Kurs von Wertpapieren bzw. Marktindizes durch einen Agenten "beobachten" zu lassen. In Abhängigkeit von der Veränderung bestimmter Kurse oder Indizes (sogenannter Benchmarks) können Agenten die eingegebenen Orders dynamisch verändern. So ist es denkbar, daß ein Limitpreis 
seitens des Agenten nach oben (unten) korrigiert wird, wenn ein entsprechender Marktindex - etwa der DAX - steigt (fällt).

Die Eigenschaft der Flexibilität ermöglicht es dem Händler, seine Agenten verschiedenartig zu konfigurieren und damit die Grundvoraussetzung für den Einsatz dynamischer Marktmodelle zu erfüllen (3). Ein konkretes Anwendungsgebiet der flexiblen Ordergestaltung betrifft die Informationen, die ein Agent von Agenten anderer Händler erhält bzw. an sie weitergibt. In Abhängigkeit vom gewählten Marktmodell kann der Agent die erhaltenen Informationen in unterschiedlichem Ausmaß an andere Agenten weitergeben. Es ist jedoch auch möglich, diese Informationen im Agenten zu kapseln, d. h. nicht weiterzugeben und lediglich zu einem Abgleich von Wertpapiereigenschaften im Rahmen der Suche zu benutzen. Auf diese Weise wird der oben erarbeiteten Anforderung, alternative Grade der Marktransparenz bereitzustellen, Rechnung getragen. So kann beispielsweise ein Blockhändler - ähnlich wie bei der Einschaltung eines Brokers - selbständig entscheiden, welche Art bzw. welche Menge an Informationen er dem Markt zeigen will.

Die Fähigkeit zur sozialen Interaktion, d. h. der Kommunikation mit dem Benutzer (4) und anderen Agenten (5), ist bei der Erfordernis einer parallelen und anonymen Suche nach Produkten und Partnern sowie bei der Preisermittlung der zentrale Vorteil des Agentenansatzes gegenüber anderen Softwareparadigmen. Bisher mußte der Händler eine Order zur Ausführung an den Kursmakler weiterleiten bzw. - im Falle des außerbörslichen Handels selbst oder durch die Zwischenschaltung von Brokern einen geeigneten Kontraktpartner finden. Diese Aufgabe kann er an einen Agenten delegieren, da Agenten insbesondere bei der Suche besonders leistungsfähig sind und diese autonom (6) durchführen können. Dabei muß der Agent einen entsprechenden Agenten als Kontraktpartner auf der Marktgegenseite finden, um dann einen Abgleich der eventuell unscharf definierten - Wertpapiereigenschaften durchzuführen.

Ist dieser Prozeß der Partner- und Produktsuche erfolgreich abgeschlossen, kann der Agent eine Auktion oder Verhandlungen über den Preis und die Menge des zu handelnden Wertpapiers autonom (7) durchführen. Dabei geht er zielorientiert (8) vor, d. h. er setzt die vom Händler vorgegebenen Strategien um.

Auf dieser Basis wird im folgenden die Konzeption und prototypische Implementierung eines dynamischen Marktmodells vorgestellt.

\section{Umsetzung eines dynamischen Marktmodells - das System AMTRAS}

Mit AMTRAs wurde ein agentenbasiertes, elektronisches Wertpapierhandelssystem entworfen und prototypisch umgesetzt. Gegenwärtig ist ein Modul für den Handel mit festverzinslichen Wertpapieren implementiert. Im 
Zuge der Systemkonzeption wurden umfassende Interviews und Befragungen durchgeführt. Dabei wurden sowohl Experten und Wertpapierhändler verschiedener Handelshäuser als auch Mitarbeiter der Deutschen Börse befragt und die Probleme des derzeitigen Handels sowie eventuelle Verbesserungspotentiale identifiziert. Während der Interviews wurde auch die Frage der schwachen Akzeptanz der gegenwärtigen elektronischen Handelssysteme im Rentenhandel erörtert (Gomber et al. 1998). Aufgrund dieser Untersuchung wurden die Anforderungen an ein elektronisches Rentenhandelssystem erarbeitet.

Dem System AMTRAS liegt ein dynamisches Marktmodell zugrunde, denn die Strukturmerkmale des Marktmodells können eine Vielzahl von Ausprägungen annehmen. Die Dynamik bei AMTRAS ergibt sich aus der Tatsache, daß ein Händler für jede Order selbständig über das Marktmodell entscheiden kann. Nahezu alle oben aufgeführten Strukturmerkmale sind miteinander kombinierbar: Es ist beispielsweise möglich, eine Kauforder mit unscharfen Couponangaben zu spezifizieren, um anschließend eine weitere Order - über ein konkretes Wertpapier mit dem Wunsch an der Teilnahme an einer Auktion abzugeben. Dabei können sich beispielsweise die Partnersuchräume, die Dauer der Ordergültigkeit und/oder der Grad an Anonymität/Markttransparenz voneinander unterscheiden.

Statt der konventionellen Orderbücher existieren auf einem elektronischen Wertpapiermarkt Agenten, die jeweils die Transaktionsinteressen der Händler repräsentieren und nach anderen Agenten, d. h. Verhandlungspartnern, mit kompatiblen Transaktionsinteressen suchen. Jeder Agent repräsentiert eine Order, die der Händler hinsichtlich der relevanten Parameter frei spezifizieren kann. Die Agenten sind beispielsweise in der Lage, einen Verhandlungspartner nach konkreten oder vagen Spezifikationen zu suchen, Auktionen durchzuführen und Verhandlungen des Händlers vollständig elektronisch - entweder nach einer vorgegebenen Strategie oder durch aktives Eingreifen des Händlers - durchzuführen.

Vor der Instantiierung der Agenten auf der Plattform muß der Händler seine Order parametrisieren, d. h. dem System seine Handelspräferenzen mitteilen. Dies geschieht durch Eingabe der Order über das AMTRAs-Frontend. Bei der Parametrisierung von Orders kann der Händler unscharfe Eingaben tätigen. Wie in Abschnitt 3.1 erläutert, muß der Händler dabei kein konkretes Wertpapier spezifizieren. Im implementierten Rentenhandelsmodul kann er Laufzeitund/oder Couponbereiche eingeben sowie die in Frage kommende Art des Papiers (Bundesanleihe, Jumbo-Pfandbrief, klassischer Pfandbrief usw.) und den Emittenten (Bund, Post, verschiedene Hypothekenbanken usw.) durch Angabe von Gruppen ähnlich unscharf spezifizieren. Auch können gewöhnliche Limitoder Marktorders eingegeben werden. Bei diesen Orders ist sowohl das Wertpapier - durch die WKN - konkret spezifiziert als auch die gewünschte Menge, die Dauer der Ordergültigkeit sowie das Limit, d.h. der höchste (niedrigste) Preis bei einer Kauforder (Verkauforder). 
Entsprechend der in Abschnitt 3.2 formulierten Anforderungen kann ein Händler alternative Suchräume individuell spezifizieren und seinen Agenten beauftragen, nur mit Agenten von Händlern dieser Suchräume zu kommunizieren. Nur wenn beide Händler sich in ihren Suchräumen gegenseitig spezifiziert haben, kommt ein Matching zustande. Die Händler können dabei individuell für jede Order entscheiden, ob - und wenn ja, in welchem Maße - sie den restlichen Marktteilnehmern Informationen über ihre Order zufließen lassen. Dabei sind nahezu alle Ausprägungen des Grades der Informationstransparenz denkbar: Angefangen von der vollständigen Anonymität bezüglich der Identität eines Händlers, einschließlich seiner Angaben über die gewünschten Volumina und Preise, über die bloße Information bezüglich der Art der Order (Kauf oder Verkauf), bis hin zur völligen Informationstransparenz sind auch Zwischenstufen der Markttransparenz einstellbar.

Nach Eingabe der Order wird ein neuer Agent auf der Handelsplattform erzeugt und die Suchphase beginnt. Der Agent sucht nach einem geeigneten Matchingpartner und einem passenden Produkt. Ein solcher Partner ist gefunden, wenn die jeweiligen Spezifikationen - also die Partnersuchräume und die Produktspezifikationen - der Agenten übereinstimmen.

Nach einem erfolgreichen Abschluß der Suchphase sind - je nach Vorgaben der Händler - zwei Möglichkeiten denkbar: Entweder erhalten die Händler die Namen bzw. Telefonnummern der gefundenen Partner und können mit diesen telefonisch über Preise verhandeln, oder es beginnt die elektronische Preisfindungsphase. Dabei sind mehrere Ausprägungen der elektronischen Preisfindung denkbar und können individuell für jeden Transaktionswunsch durch den Händler festgelegt werden. Gegenwärtig sind im System AMTRAS folgende Ausprägungen implementiert (Gomber et al. 1998):

- Auktionen und

- Verhandlungen mit

(a) verbindlichen und

(b) unverbindlichen Orders.

Als Auktion sieht das Marktmodell von AMTRAS eine Single auction vor. Dabei trifft die eingestellte Order auf eine oder mehrere Orders auf der Marktgegenseite. Zur Teilnahme an einer solchen Auktion müssen die Händler konkrete Wertpapiere, das gewünschte Volumen sowie einen Limitpreis spezifizieren.

AMTRAS ermöglicht auch die Abbildung elektronischer Verhandlungen. Für diesen Zweck müssen die Händler neben dem Wertpapier auch die von ihnen gewünschten maximal zu handelnden Volumina angeben. Anschließend können die Händler in einem Preis/Zeit-Diagramm ihre Handelspräferenzen in Form einer Funktion - der sog. Verhandlungsfunktion - angeben. Dabei handelt es sich um eine diskrete Funktion, die jedem Verhandlungszeitpunkt genau einen Limitpreis zuordnet. Diese Funktion kann als Verhandlungsstrategie eines Händlers interpretiert werden. Die einzelnen Zeitabstände (Ticks) sind dabei vom 
System fest vorgegeben. Findet nun ein Produkt- und Partnermatching statt, kommt es zu einer elektronischen Verhandlung. Die Verhandlungsfunktionen der Händler werden nun gegenübergestellt und bei jedem Tick miteinander verglichen. Wesentlich hierbei ist die Möglichkeit des aktiven Eingreifens der Händler in die laufenden Verhandlungen. Die Händler können jederzeit ihre Verhandlungsfunktionen ändern und somit - wie in den heutigen außerbörslichen, telefonischen Verhandlungen - interaktiv und in Echtzeit miteinander über den Preis des Wertpapiers verhandeln.

Wählt der Händler im Marktmodell die Möglichkeit der verbindlichen Verhandlungen, so erfolgt nach einer erfolgreichen Verhandlung und Preisfindung mit seinem Kontraktpartner ein sofortiger, automatischer Abschluß des Geschäfts. Bei einer unverbindlichen Verhandlung hingegen folgt nach der erfolgreichen Preisfindung nur eine Bekanntgabe der Verhandlungsergebnisse an die Händler. Um zu einem Abschluß zu gelangen, müssen beide Händler das Verhandlungsergebnis der Agenten bestätigen.

AMTRAS wurde vollständig objektorientiert modelliert und in der Programmiersprache Java implementiert, so daß eine weitgehende Plattformunabhängigkeit erreicht wurde (Lohmann et al. 1998). Aufgrund der objektorientierten Modellierung des Systems ist eine Erweiterung um weitere Marktsegmente, wie etwa den Aktien- oder Derivatehandel möglich. Die Architektur wurde auf Basis des FIPA-Standards konzipiert (FIPA 1997). Als Grundlage für das Kommunikationsprotokoll wurde TCP/IP gewählt.

Die Agenten "leben" und kommunizieren auf einer zentralen Serverplattform. Diese Lösung wurde gewählt, um sicherzustellen daß die Plattform stets unter Aufsicht einer zentralen Instanz - z.B. einer Börsenorganisation - operieren kann. Dadurch wird die Sicherheit und damit das Vertrauen der Marktteilnehmer gewährleistet (Weinhardt und Gomber 1998). Der Server ist beliebig skalier- und erweiterbar, so daß bei Bedarf - etwa bei hoher Systemauslastung - während der Laufzeit weitere Plattformen hinzugefügt werden können. Zur Gewährleistung der Stabilität bzw. der Verfügbarkeit des Systems gibt es keinerlei dedizierte Komponenten.

Die Clients sind gleichzusetzen mit Einzelplatzrechnern der Wertpapierhändler. Auf dem Client befindet sich eine grafische Benutzeroberfläche, das sog. AMTRAS-Frontend, über die der Händler alle Eingaben tätigt und die Handelsergebnisse gemeldet werden. Diese Eingaben werden an die Plattform übermittelt, so daß der Client als pures Ein- und Ausgabegerät zu sehen ist und somit nicht als Bestandteil der Agentenplattform gilt. 


\section{Zusammenfassung und Ausblick}

In der Arbeit wurde das Konzept dynamischer Marktmodelle motiviert und gegen die bisher existierenden statischen und flexiblen Marktmodelle für den Wertpapierhandel abgegrenzt. Dynamische Marktmodelle zeichnen sich dadurch aus, daß der Händler individuell über die jeweiligen Ausprägungen der Handelsparameter entscheiden kann. Im Extremfall kann für jede eingegebene Order ein anderes Marktmodell festlegt werden. Damit kann man auf einer Handelsplattform den heterogenen Anforderungen an Marktmodelle - z.B. dem Grad der Markttransparenz oder der Art der Preisfeststellung - gerecht werden.

Die Umsetzung dieses Konzepts erfolgte im System AMTRAS auf Basis der Technologie der Softwareagenten. Gegenwärtig existiert ein Modul für den Rentenmarkt. Da das System vollständig objektorientiert modelliert und implementiert wurde, ist eine Erweiterung auf weitere Marktsegmente, wie etwa den Aktien-, Devisen- oder den Markt für verschiedene derivative Finanzinstrumente, möglich.

Die Weiterentwicklung des Systems für andere Marktsegmente, die Integration weiterer Auktions- und Verhandlungsmechanismen und insbesondere die Untersuchung des implementierten Marktmodells bzw. der Leistungsfähigkeit des Systems (Stabilität und Performance bei großen Teilnehmerzahlen) stehen im Vordergrund weiterer Forschungsvorhaben. Neben Interviews und Befragungen zur Akzeptanz seitens der Händler soll auch die Methodik des wirtschaftswissenschaftlichen Experiments (Gerke und Bienert 1994) Anwendung finden. Dabei können innovative Marktmodelle bezüglich ihrer Vor- und Nachteile im Vergleich zu den aktuellen Handelsformen beurteilt werden.

Mit der zunehmenden Akzeptanz und Verbreitung Elektronischer Märkte gewinnen auch bei Gütermärkten - neben der traditionellen Preissetzung durch den Produktanbieter - verstärkt Auktionen und Verhandlungsmechanismen an Bedeutung. Auch werden Fragen der Markttransparenz über Wertpapiermärkte hinaus relevant, etwa bei Elektronischen Märkten zur Vermittlung von Arbeitsplätzen. Die Erkenntnisse des Ansatzes eines dynamischen Marktmodells und die Umsetzung des Konzepts mit dem Agentenparadigma wird damit auch einen Beitrag zur Gestaltung von innovativen Elektronischen Güter- und Dienstleistungsmärkten liefern.

\section{Literaturverzeichnis}

Braue, C./Hille, L. (1997): Xetra - Elektronisches Handelssystem am Finanzplatz Deutschland. Die Bank, (1997)3, S. 140-145. 
Brunner, A. (1996): Meßkonzepte zur Liquidität auf Wertpapiermärkten. Beiträge zur Theorie der Finanzmärkte Nr. 13. Herausgegeben vom Institut für Kapitalmarktforschung, Frankfurt am Main, 1996.

Büschgen, H. E. (1993): Bankbetriebslehre: Bankgeschäfte und Bankmanagement. Vierte, vollständig überarbeitete und erweiterte Auflage. Wiesbaden, Gabler, 1993 (1. Auflage 1973).

Deutsche Börse (1998a): Erster Schritt zu einem einheitlichen europäischen Aktienmarkt. Reporter. Newsletter der Deutschen Börse. Juli 1998, S. 1.

Deutsche Börse (1998b): Xetra ${ }^{\circledR}$ : Marktmodell Release 3 Aktienhandel. 1998.

Deutsche Bundesbank (1995): Die Geldpolitik der Bundesbank. Oktober 1995.

Easley, D./O'Hara, M. (1992): Adverse Selection and Large Trade Volume: The Implications for Market Efficiency. Journal of Financial and Quantitative Analysis, 27(1992)2, pp. 185-208.

FIPA (1997): Foundation for Intelligent Physical Agents (FIPA): FIPA 97 Specification, Version 1. http://drogo.cselt.stet.it/fipa/spec/fipa97/fipa97. htm, 1997.

Gerke, W. (1993): Computerbörse für den Finanzplatz Deutschland. Die Betriebswirtschaft, 53(1993)6, S. 725-748.

Gerke, W./Bienert, H. (1994): Computerisierte Börsenexperimente: Ein Beitrag zu einer Experimentellen Kapitalmarktforschung. Zeitschrift für Wirtschafts- und Sozialwissenschaften (ZWS), 114(1994)4, S. 573-596.

Gerke, W./Rapp, H.-W. (1994): Strukturveränderungen im internationalen Börsenwesen. Die Betriebswirtschaft, 54(1994)1, S. 5-23.

Gerke, W./Rasch, S. (1992): Ausgestaltung des Blockhandels an der Börse. Die Bank, (1992)4, S. 193-201.

Gomber, P. (1998): OptiMark - ein neues Handelssystem in den USA. Die Bank (1998)11, S. 682-684.

Gomber, P./Budimir, M./Kosciankowski, K./Urtheil, R. (1998): Elektronisierung des außerbörslichen Rentenhandels auf Basis von Softwareagenten. In: Weinhardt, C./Meyer zu Selhausen, H./Morlock, M. (Hrsg.): Informationssysteme in der Finanzwirtschaft. Heidelberg et al., Springer, 1998, S. 253-267.

Hasbrouck, J./Schwartz, R. A. (1988): Liquidity and Execution Costs in Equity Markets. Journal of Portfolio Management, (1988) Spring, pp. 10-16.

Lohmann, M./Nopper, N./Henning, P. (1998): Elektronisches Kontrahentenmatching im agentenbasierten Wertpapierhandel. In: Weinhardt, C./Meyer zu Selhausen, H./Morlock, M. (Hrsg.): Informationssysteme in der Finanzwirtschaft. Heidelberg et al., Springer, 1998, S. 269-284. 
Lüdecke, T. (1996): Struktur und Qualität von Finanzmärkten. Wiesbaden, Gabler, 1996. Zugleich: Diss., Universität Karlsruhe 1995.

Luskin, D. L. (1988): Upstairs, Downstairs: The Block Traders and the Specialist. In: Wagner, W. H. (Ed.): The Complete Guide to Securities Transactions. New York, John Wiley and Sons, 1988, pp. 153-160.

Maes, Pati (1995): Artificial Life Meets Entertainment: Life Like Autonomous Agents. Communications of the ACM 38(1995)11, pp. 108-114.

Modigliani, F./Fabozzi, F. J. (1992): Capital Markets: Institutions and Instruments. Englewood Cliffs (NJ), Prentice-Hall, 1992.

o. V. (1998): Die DTB heißt jetzt Eurex. Handelsblatt, Nr. 115, 19./20. Juni 1998. S. 25.

Thießen, F. (1995): Wer braucht die Frankfurter Devisenbörse? Die Bank (1995)1, S. 15-19.

Weinhardt, C./Gomber, P. (1998): Elektronisierung des außerbörslichen Wertpapierhandels. Konzeption und Engineering eines finanzwirtschaftlich und mikroökonomisch basierten Multi-Agenten-Ansatzes. In: Wirtschaftsinformatik, 41(1999)1.

Wooldridge, M. J./Jennings, N. R. (1995): Intelligent Agents: Theory and Practice. The Knowledge Engineering Review, 10(1995)2. 